DOI: $10.22363 / 2313-2302-2017-21-1-17-23$

\title{
MORAL UND RELIGION IN DER PHILOSOPHIE VON WOLFF
}

\author{
L.E. Kryshtop \\ Peoples' Friendship University of Russia (RUDN University) \\ Miklukho-Maklaya Str., 6, Moscow, Russia, 117198
}

\begin{abstract}
Christian Wolff, a one of the most well-known philosophers of the first half of the $18^{\text {th }}$ century, is the figure quite ambiguous. In spite of the fact, that he is usually ranged chronologically among the thinkers of Enlightenment, there are researchers which find in his philosophical views some features inherent more to the scholastic tradition. But there are also the features which demonstrate Wolff as the thinker of Enlightenment. At the first it is Wolff's striving to praise the human reason and its capacities, that comes together with the fight for its liberation and independence, among all from God. We can show it with the greatest evidence in his practical philosophy, in which he tries to deduce the natural law, which he hold for the first principle of the morality, from the human nature and to give it the justification independent from God and His will.
\end{abstract}

Key words: Wolff, morality, religion, natural law, perfection, good, evil, virtue, God, nature, reason, understanding, will

Ein von den deutschen bekanntesten Philosophen der ersten Hälfte des 18. Jahrhunderts, seiner Zeit hochgepriesener, jetzt aber leider kaum nicht nur die Fachkenner interessierender Christian Wolff (1679-1754) ist sowohl hervorragender, als auch umstrittener Denker. Er lebte und arbeitete in der Zeit, die man gewöhnlich als der Zeitalter der Aufklärung in Deutschland bezeichnet (1), trotzdem ist die Frage, ob wir ihn zu aufklärerischem Philosoph nennen können, bis heute in Forschungsliteratur heiß diskutiert [6, S. 306-307; 14, S. 3-4; 12, S. 14-15; 8, S. 109; 4, S. 35-37].

Es gibt aber nicht wenige Gründe, Wolff zu den Philosophen der Aufklärung hinzurechnen (obwohl vielleicht mit gewissen Einschränkungen). Alle Art der Aufklärung (der Nationalität ungeachtet) verlangte nach Erhebung der Vernunft und Reinigung von den verschiedenen Vorurteilen und Irrtümer in allen Bereichen des menschlichen Lebens. Worin soll die Reinigung bestehen? Was soll zu den Vorurteilen und Irrtümer zugeordnet werden? Auf welcher Weise war die Reinigung durchzuführen? An die Fragen können wir schon in verschiedenen Ländern bei verschiedenen Denkern verschiedene Antworten finden. Aber für alle Aufklärer waren sowohl die feste Orientierung an die Vernunft und die Hervorhebung ihrer ausgezeichnete Wichtigkeit für die moralische Entwicklung des Menschen, als auch die Versuche, sie von allem irreführenden und falschen zu reinigen, charakteristisch. Das war auch für Wolff geeignet, was wir vor allem in seiner praktischen Philosophie spüren können.

Wolff formuliert die Hauptprinzipien von seinem ethischen System schon in „Deutscher Ethik“, die 1720 erschienen wurde. Bereits da finden wir zwei grundlegende Begriffe von der praktischen Philosophie Wolffs, nämlich den Begriff von Vollkommenheit und den von Naturgesetz. 
Der Begriff von Vollkommenheit ist mit dem Begriff von Gut eng verbunden. Das Gut ist das, was jeder Mensch erreichen will. Dabei bestiemt Wolff das Gut als das, „was unseren so wohl innerlichen, als auch äusserlichen Zustand vollkommen machet" [18, S. 6], vgl. [19, S. 260]. Das Gegenteil des Guten — das Böse — ist im Gegenteil das, was uns und unsere Zustände unvollkommener macht [18, S. 6; 19, S. 262; 17, S. 391]. So wird Vollkommenheit zu dem Hauptkriterium, auf Grunde dessen wir urteilen, ob Dinge, Zustände und Handlungen gut oder böse sind. Alle unsere Handlungen, die dazu beitragen, dass die Vollkommenheit der Welt sich vermehrt, sind gut, und die, die sie vermindern, sind an sich selbst schlecht [18, S. 6-7]. Dabei hält Wolff die Vollkommenheit für kein statisches Idealzustand, das einem Ding zukommt, sondern spricht über verschiedenen Stufen der Vollkommenheit, denn je harmonischer die Übereinstimmung der Zustände von einem Ding ist, desto vollkommener ist es. Infolgedessen wird einen Fortschritt für uns möglich, ein Streben nach der Vermehrung der Vollkommenheit sowohl in uns selbst, als auch in der Umgebung. Und nur dies unendlicher Fortschritt zu einer immer größeren Vollkommenheit ist unser Schicksal, denn die höchste Vollkommenheit kann nur bei Gott sein und keine von seinen Schöpfungen (eben deswegen, weil sie von Gott geschöpft ist) imstande ist, sie zu erreichen [18, S. 31; 19, S. 671]. Aber diese Vergrößerung der Vollkommenheit in der Welt (inwiefern es unter unserer Gewalt steht) muss die Hauptabsicht von dem ganzen unserem Leben sein. Alle anderen besonderen Absichten in sich selbst und in ihrem Ganzen müssen unter dieser Hauptabsicht als die Mittel, sie zu erreichen, zugeordnet werden [18, S. 29-30, $79-80,84-85]$.

Was treibt aber einen Menschen an, die Vollkommenheit zu seinem Ziel zu machen? Genauere Betrachtung dieser Frage führt Wolff zur Formulierung der Hauptregel von seiner Moral, die er als Naturgesetz bezeichnet hat. Nach den allgemeinen ontologischen Gründen von allen Dingen der Welt gesucht kommt er zu der Schlussfolgerung, dass wir sie nirgendwo anders zu suchen haben, als nur in den Dingen selbst, in ihren Wesen. Denn alles, was passiert, die Gründe des Daseins entweder in sich selbst (in eigenem Wesen) oder in etwas Äußerliches (im Wesen eines anderen Dings) haben muss. Also liegen die Gründe von allem passierenden allerdings in den Dingen selbst. Im Fall der freien Handlungen der Menschen sind ihre Gründe in der Natur der menschlichen Seele zu suchen. Der Schluss, den Wolff daraus zieht, ist folgender: alle unsere freie Handlungen resultieren sich aus unserer eigenen Natur und aus der Natur der Dinge, aus ihrer Beschaffenheit. Aus unserer Natur ergeben sie sich deswegen, weil die Vorstellungen darüber, was gut ist und was schlecht ist, sich in unserer Seele befinden. Aus der Beschaffenheit der Dinge ergeben sie sich, denn diese Dinge an sich selbst entweder gut oder böse sind. Sind unsere Vorstellungen von dem Gut und dem Böse richtig, d.h. unsere Vorstellungen von den Dingen den tatsächlichen Dingen entsprechen, so machen wir gute Handlungen. Irren wir uns entweder in Bezug darauf, was gut und was böse ist, oder in Bezug auf das Wesen der Dinge, so machen wir böse Handlungen. Also die Natur der Dinge und unsere eigene Natur verpflichten uns das Gut zu wollen und das Böse zu vermeiden [18, S. 9-10] und auch das, was besser ist, voranzuziehen [18, S. 10]. Oder mit dem anderen Wort das zu unternehmen, was unsere innere und äußere Zustände vollkommener macht, und das zu vermeiden, was sie unvollkommener macht [18, S. 11-12; 16, S. 127]. Daraus schließt Wolff die allgemeine Regel, die 
lautet: „Thue das, was dich und deinen oder anderer Zustand vollkommener machet: unterlaß, was ihn unvollkommener machet" [17, S. 12], vgl. [15, S. 21-22; 16, S. 113]. Diese Regel ist gleichzeitig ein Gesetzt, denn man nennt als Gesetzt „eine Regel, darnach wir verbunden sind, unsere freye Handlungen einzurichten“ [18, S. 15], und genau von der Art ist die oben genannte Regel. Und da dieses Gesetzt sich unmittelbar aus der Natur der Dinge ergibt und seinen Grund in der Natur von unserer Seele hat, wird es von Wolff als Naturgesetzt bezeichnet $[18, \mathrm{~S} .15,16]$. Diese Regel greift sich ausnahmslos um alle freie menschliche Handlungen und ist darum allgemein, was heißt, dass alle andere besondere Regeln daraus gezogen werden müssen: „Da nun diese Regel sich auf alle freye Handlungen der Menschen erstrecket; so hat man kein anderes Gesetze der Natur mehr nöthig, sondern alle besondere Gesetze müssen daraus erwiesen werden" [18, S. 16], vgl. [18, S. 13, 19, 24-26]. Also steht alles, was gut ist, d.h. was uns vollkommener macht, in Übereinstimmung mit unserer Natur und umgekehrt, was unserer Natur entspricht, ist gut. Das beste Mittel, die Glückseeligkeit zu erreichen, ist also nichts anderes, als beständige Einhaltung des Naturgesetzes [18, S. 32, 35]. Die Bereitschaft auf dieser Weise im Leben zu handeln nennt Wolff Tugend [18, S. 41] und behauptet, dass niemand glückselig sein kann, als nur ein tugendhafter Mensch [18, S. 43], denn nur auf dem Weg erreicht man das höchste Gut, das Wolff als einen beständigen unendlichen Fortschritt zur Vollkommenheit definiert.

In dem Stück von den Aussichten Wolffs verbergen sich gerade die Gründe, Wolff Atheismus und Freidenkerei anzuklagen, was zur Folge hatte, dass er 1723 aus Halle verbannt wurde. Denn wenn es reicht, um Glückseligkeit zu erreichen das Naturgesetz allein zu befolgen, das zwar mit dem Willen Gottes übereinstimmt, hängt aber unmittelbar davon nicht, infolgedessen kann auch ein Atheist wohl glückselig werden, so bedeutet das denn nicht, dass Wolff Gott selbst unter dem Naturgesetz zu ordnen suchte? Eine Handlung ergibt sich als gute nicht deswegen, weil sie Gott gefällt, sondern ganz umgekehrt die Handlung gefällt Gott, denn sie ist gut und Gott kann aus der Beschaffenheit von seiner Natur nichts anderes wollen, als nur das bloße Gut [19, S. 607608]. So können wir den Schluss, ob eine Handlung gut oder böse ist, auf Grunde unseres Erkenntnisses vom Naturgesetz und noch vor dem, als wir das Dasein Gottes erkennen und bewiesen haben, machen. Denn die Handlungen sind gut oder böse an sich selbst und nicht durch Gottes Willen und Gefallen: „Wenn es derowegen gleich möglich wäre, daß kein Gott wäre, und der gegenwärtige Zusammenhang der Dinge ohne ihn bestehen könnte; so würden die freyen Handlungen der Menschen dennoch gut oder böse verbleiben" [18, S. 7].

Ein unumstrittener Vorteil dieser Sichtsweise ist es, dass auch Atheisten sich amoralisch verhalten nicht dürfen, weil die Regeln der Moral sich aus der Natur ziehen lassen und von dem Willen Gottes unabhängig zu sein scheinen [18, S. 17] (2). Aber dieser Schluss hat auch einen Nachteil. Wenn das Naturgesetz höher als Gott und Gottes Wille steht und Gott als unter dem Naturgesetz geordneter zu betrachten ist, so bedürft ein vernünftiger Mensch nichts außer den natürlichen Pflichten, um glückselig zu werden [18, S. 18, 28-29]. Anders gesagt handelt ein vernünftiger Mensch dem Naturgesetz gemäß und braucht keine andere Gesetze (sowohl bürgerliche als auch göttliche), denn die Vernunft wird in dem Fall für sich selbst ein Gesetz [18, S. 18]. Und nur dadurch laut 
Wolff kann ein Mensch zu Gott ähnlich wird, denn Gott hat niemand über sich, der Gott etwas befehlen könnte, und trotzdem macht er das Gut aus der Vollkommenheit seiner Natur [18, S. 28-29]. Zu dem ist auch jeder Mensch bestimmt.

Dieser Schluss von Wolff gibt M. Albrecht den Grund zu behaupten, dass es Wolff gelungen hat, praktische Philosophie von der theologischen Begründung zu befreien und sie zum Grunde die autonome menschliche Vernunft zu legen [1, XXXVI]. An die Unabhängichkeit der Moral von der Theologie in Wolffs Philosophie zeigt auch W. Schneiders, der aber in dem Bezug vorsichtiger ist und diese Unabhängichkeit nur als stark begrenzte charakterisiert [11, S. 158]. Und in der Tat, obwohl es sich von dem ersten Blick erscheinen kann, dass das von Wolff als Hauptregel der Moral angebotene Naturgesetz sich ganz unabhängig von Gott erkennen lässt, erweist es sich bei der präziseren Betrachtung, dass die Notwendigkeit Gott ins philosophischen System einzutragen und die Wichtigkeit des christlichen Glaubens zu begründen sich nicht nur episodischerweise ergibt, wenn Wolff die besondere menschliche Pflichten gegen sich selbst und Gott untersuchen will, sondern seine Wurzeln viel tieferer hat und zwar in der Notwendigkeit die Hauptbegriffe der praktischen Philosophie Wolffs - Naturgesetzt und Vollkommenheit — zu begründen [14, S. 5].

Das Naturgesetz wird von Wolff als schlechterdings notwendig betrachtet, denn das zum menschlichen Wesen geeignet ist. Wir Menschen sind solche Kreaturen, dass die Regel von Moral und ihre Einhaltungen für uns natürlich sind, egal ob wir es mögen oder nicht. Da hängt nichts von unserem Erkenntnis dieses Gesetzes und von unserer Bereitschaft es für Wahrheit zu halten ab. Jedenfalls richtet der Mensch seine Handlungen diesem Gesetz gemäß ein. Solche Unterstreichung der Tatsache, dass das Naturgesetzt in der Natur des menschlichen Wesens eingewurzelt ist (3), kann den irreführenden Eindruck machen, als ob es Wolff gelungen hat, seine Hauptregel der Moral unabhängig von Gott zu deduzieren, und als ob seine Ethik sich als naturalistisch erweist.

Aber im Hintergrund von diesen Thesen steht die andere Frage, und nämlich: woher kommt solchergestalt geschöpfte Natur des Menschen? Und evidenterweise ergibt sich die Antwort: Gott hat die Natur des Menschen so beschaffen geschöpft. Und warum hat Gott sie so beschaffen geschöpft? Denn solcher Lauf der Dinge ist der beste und der vollkommenste aus allen möglichen. Diese Welt auf der Weise schaffen kann Gott nur aus der Vollkommenheit von seiner Natur. Und diese Vollkommenheit muss sowohl intellektuelle (die Vollkommenheit des Verstandes, die ihm wissen und bestimmen lässt, was gut und vollkommen ist) als auch moralische (die Vollkommenheit des Willens, die ihm immer nur das Gute wollen lässt) Vollkommenheit sein. Und so erweist sich Gott bei Wolff als Allvollkommenes, Allgütiges, Allwissendes, Allmächtiges und s. w. Wesen, ganz seinem traditionellen Begriff gemäß. Und solches Höchste Wesen tritt bei Wolff als Garant für die Natur des Menschen und der Welt, aus denen sich das Naturgesetz ziehen lässt, auf. Also wir können sagen, dass es Wolff gelungen hat, die Theonomie in der Begründung der besonderen ethischen Vorschriften zu vermeiden, denn sie alle durchs Naturgesetz aus dem Wesen der menschlichen Seele und der Natur der Dinge und nicht aus dem Willen Gottes gezogen werden. Aber erstaunlicherweise erweist sich diese atheonome Begründung als möglich nur dank Gott, dem Allmächtigen, der so vollkommen ist, um imstande zu sein, diese Welt so vollkommen zu schaffen, dass seine weitere Einmischung mehr unnötig und einfach entbehrlich ist. 
Von diesem Standtpunkt aus erscheint das ganze Ethiksystem von Wolff in einem anderen Licht. Auch in den Fälle, wenn der Denker direkt sagt, dass der Glaube an Gott nicht nötig ist, um ein tugendhafter Mensch zu werden, unterstürzt und gerechtfertigt er damit Atheisten gar nicht, sondern versucht zu erweisen, dass auch die Menschen, die egal von welcher Ursachen des wahren Erkenntnissen Gottes und seiner Beschaffenheiten beraubt sind, kein Recht haben, sich amoralisch zu verhalten, denn alle Leute unabhängig von ihrem religiösen Glaubensbekenntnis unter demselben Naturgesetz zugeordnet sind. Also es ist korrekter über Wolff als kein Verteidiger von Atheisten, sondern als ihr Ankläger zu reden, der prinzipielle Inkonsistenz und Widersprüchlichkeit in ihren Verwendung des Verstandes aufzudecken versuchten, was unter anderen M. Pott in seiner Arbeit, die der aufklärerischen Vorurteils- und Aberglaubenskritik gewidmet ist, macht [9, S. 184 -186], vgl. [7, S. 208; 10, S. 406]. Denn die wahre Moral ist bei Wolff ohne Gott nicht zu realisieren und auch nicht zu denken.

\section{SCHLUSS}

Nachdem wir die Hauptprinzipien der Moralphilosophie Wolffs und unmittelbar damit verbundene Ansichten von dem höchsten Wesen in Betrachtung gezogen haben, können wir beschließen, dass trotz der scheinbaren atheonomen und atheistischen Begründung von Wolffs Ethiksystem, gelingt es Wolff nicht die Aufstellung und Besprechung von der ganzen Reihe der Fragen zu vermeiden, die Bezug auf Gott und ihm zugeschriebene Prädikate haben. Die Lösung von den Fragen ergibt sich als äußerst wichtige für das ganze philosophische System von Wolff sowohl in seinem theoretischen als auch praktischen Teil. Und das alles, des Wolff geeigneten aufklärerischen Anspruch, alles vernünftig zu erklären und keine Voraussetzungen ohne zureichende Begründung anzunehmen, ungeachtet führt uns doch zu der schon seit dem Mittelalter gut bekannten Tradition, Gott als der Grund des Daseins von der ganzen Welt und der Urprinzip von unseren möglichen Erkenntnissen zu bezeichnen und ihm die Prädikate zulegen, die unsere Vernunft überschreiten und ihn über unsere Logik erheben.

Gleichzeitig wäre es eher unkorrekt, die aufklärerischen Leistungen von Wolff zu bloßer äußerlichen Schein und äußerlichen ganz formalen Bestrebungen alles unter den Gesetzen der Vernunft zu ordnen, die wegen der tieferen Wurzeln, die Wolff in der scholastischen Philosophietradition geschlagen hat, was unmöglich macht, die solche unbegründete Voraussetzungen zu bemerken und sie in Frage zu stellen, unvermeidbar zu Bruch gehen. Denn Wolffs Bestrebung alles mit der Hilfe der mathematischen Methode zu untersuchen führt ihn endlich zur Notwendigkeit, Gott als ein Gegenstand von wissenschaftlicher Betrachtung auch zu den ausgearbeiteten Regeln von Rationalität zu ordnen. Freilich bringt die konsequente Entwicklung von dieser Aussicht zu den widersprüchlichen Schlussfolgerungen, denn der Glaube an Gott (und nicht nur an Gott von Philosophen, sondern an dreifaltigen Gott von Christentum) ist zwar nicht unentbehrlich für Tugend, aber unentbehrlich, um die höchste Grad derselben zu erreichen, und die verteidigte von Wolff Autonomie und Selbstständigkeit der menschlichen Vernunft ist nur aufgrunde Gott als dem letzten und übervernünftigen Urgrund zu erkennen [14, S. 31]. Aber trotz diesen unvermeidbaren Widersprüche [2, S. 136-137; 5, 
S. 142 - 144], wurde die Frage über die Autonomie der menschlichen Vernunft in der Philosophie Wolffs klar und deutlich gestellt, was stimmte mit dem Geist der Aufklärung völlig ein. Und genau das hat den Spielraum für weitere Untersuchungen eröffnet $[5$, S. $139,144-145]$.

\section{BEMERKUNGEN}

(1) Es ist üblich die deutsche Aufklärung gegen Ende des 17. Jahrhunderts, mit dem Beginn des akademischen Wirkens von Chr. Thomasius anzufangen [3, S. 15; 11, S. 85; 13, S. 4].

(2) An diesem und anderen ähnlichen Textstellen beruhen manche Forscher die Meinung, dass Wolff Denker war, der als der erste die konsequente Begründung der atheistischen Moralphilosophie vorgeschlagen hat $[1, \mathrm{~S} . \mathrm{XXXVI}]$.

(3) Freilich begrenzt Wolff den Geltungsbereich von diesem Gesetzt mit den menschlichen Wesen, sondern behauptet, dass alle Dinge der Welt unter ihm geordnet sind, weshalb es für den ganzen Weltraum geeignet ist, nach die möglichst große Vollkommenheit zu streben. Genau das macht die allgemeine Übereinstimmung möglich.

\section{REFERENCES}

[1] Albrecht M. Einleitung. Wolff Chr. Rede über die praktische Philosophie der Chinesen. LateinDeutsch, Hrsg. von M. Albrecht. Hamburg, 1985. S. IX_-LXXXIX.

[2] Casula M. Die Theologia naturalis von Christian Wolff: Vernunft und Offenbarung. Christian Wolff. 1679-1754. Hamburg, 1986. S. 129-138.

[3] Ciafardone R. Einleitung. Die Philosophie der deutschen Aufklärung. Texte und Darstellung. Hrsg. von R. Ciafardone. Stuttgart, 1990. S. 11-38.

[4] Frängsmyr T. The Mathematical Philosophy. The Quantifying Spirit in the 18th Century. Ed. by T. Frängsmyr, J.L. Heilbron, R.E. Rider. Berkley, Los Angeles, Oxford, 1990. P. 38 - 44.

[5] Gawlick G. Christian Wolff und der Deismus. Christian Wolff. 1679-1754. Hamburg, 1986. S. $139-147$.

[6] Hinske N. Wolffs Stellung in der deutschen Aufklärung. Christian Wolff 1679—1754. Hamburg, 1986. S. 306-319.

[7] Mauthner F. Der Atheismus und seine Geschichte im Abendlande. Happenheim, 2010.

[8] Mittelstrass J. Neuzeit und Aufklärung. Studien zur Entstehung der Neuzeitlichen Wissenschaft und Philosophie. Berlin, New York, 1970.

[9] Pott M. Aufklärung und Aberglaube: die deutsche Frühaufklärung im Spiegel ihrer Aberglaubenskritik. Tübingen, 1992.

[10] Schmidt J. Geschichte des geistiges Lebens in Deutschland von Leibniz bis auf Lessing's Tod. Leipzig, 1862.

[11] Schneiders W. Aufklärung und Vorurteilskritik. Studien zur Geschichte der Vorurteilstheorie. Stuttgart-Bad Cannstatt, 1983.

[12] Schneiders W. Die wahre Aufklärung. Zum Selbstverständnis der deutschen Aufklärung. Freiburg, München, 1974.

[13] Schneiders W. Vernunft und Freiheit. Christian Thomasius als Aufklärer. Studia leibnitiana, 1979. Bd. 11. H. 1. S. 3-21.

[14] Theis R. La question du fundement chez Christian Wolff. Theis R. De Wolff à Kant. Études. Hildesheim, Zürich, New York, 2013. P. 3-33.

[15] Wolff Chr. Institutiones juris naturae et gentium in quibis ex ipsa hominis natura continuo nexu omnes obligationes et jura omnia deducuntur. Halle, 1763. 
[16] Wolff Chr. Philosophia practica universalis, methodo scientifica pertractata. Halle, 1744.

[17] Wolff Chr. Philosophia prima sive Ontologia, methodo scientifica pertractata. Frankfurt, Leipzig, 1736.

[19] Wolff Chr. Vernünfftige Gedancken von der Menschen Thun und Lassen, zu Beförderung ihrer Glückseeligkeit. Halle, 1743.

[19] Wolff Chr. Vernünftige Gedanken von Gott, der Welt und der Seele des Menschen, auch allen Dingen überhaupt. Halle, 1747.

DOI: $10.22363 / 2313-2302-2017-21-1-17-23$

\title{
МОРАЛЬ И РЕЛИГИЯ В ФИЛОСОФИИ ВОЛЬФА
}

\author{
Л.Э. Крыштоп \\ Российский университет дружбы народов \\ ул. Миклухо-Маклая, 6, Москва, Россия, 117198
}

\begin{abstract}
Христиан Вольф - один из известнейших немецких философов первой половины XVIII в. является фигурой весьма неоднозначной. Несмотря на то, что его хронологически принято относить к мыслителям эпохи Просвещения, ряд исследователей усматривают в его творчестве черты, свойственные скорее предшествующей схоластической традиции. Однако немало черт сближает Вольфа и с просветительской традицией. И самой главной такой чертой является возвеличивание человеческого разума и его способностей, сопряженное с борьбой за его независимость и самостоятельность, в том числе и от Бога. Ярче всего это прослеживается в Вольфовой морали, когда Вольф пытается дать вводимому им в качестве основного правила морали естественному закону обоснование, совершенно не зависимое от Бога.
\end{abstract}

Ключевые слова: Вольф, мораль, религия, естественный закон, совершенство, благо, зло, добродетель, Бог, природа, разум, рассудок, воля 